\title{
Pleiotropic actions of amiodarone: still puzzling after half a century
}

\author{
Jordi Heijman • Dobromir Dobrev
}

Received: 26 March 2013 /Accepted: 31 March 2013 /Published online: 9 April 2013

(C) Springer-Verlag Berlin Heidelberg 2013

Amiodarone was developed in the early 1960s based on extracts from the Khella plant and was initially used as a coronary dilator for the treatment of angina pectoris (Singh 2008). In 1970, Singh and Vaughan Williams discovered that amiodarone also directly affected myocardial tissue, prolonging the cardiac action potential and protecting against ouabain-induced arrhythmias in guinea pigs (Singh and Vaughan Williams 1970). To date, amiodarone remains one of the most commonly prescribed antiarrhythmic drugs in clinical practice and is used in the treatment of both ventricular and supraventricular arrhythmias. In particular, amiodarone plays a major role in the maintenance of sinus rhythm in patients with atrial fibrillation (Heijman et al. 2013; Zimetbaum 2012).

The seminal Cardiac Arrhythmia Suppression and Survival Trial (CAST) and Survival with Oral D-Sotalol (SWORD) trial have highlighted the proarrhythmic risks of classes I and III antiarrhythmic drugs (Echt et al. 1991; Waldo et al. 1996). In contrast to many other antiarrhythmic drugs, amiodarone is generally not associated with drug-induced "torsades des pointes" arrhythmias or an increase in cardiovascular mortality compared to placebo (Singh 2008; Zimetbaum 2012). However, its clinical application is severely limited by its pronounced extra-cardiac toxicity. In particular, amiodarone has been associated with hepatic toxicity, manifesting as

J. Heijman · D. Dobrev $(\bowtie)$

Institute of Pharmacology, Faculty of Medicine,

University Duisburg-Essen, Hufelandstrasse 55,

45122 Essen, Germany

e-mail: dobromir.dobrev@uk-essen.de

D. Dobrev

Division of Experimental Cardiology, Medical Faculty Mannheim, Heidelberg University, Mannheim, Germany

D. Dobrev

DZHK (German Centre for Cardiovascular Research),

partner site Heidelberg/Mannheim, Mannheim, Germany transaminase elevation, thyroid dysfunction, and pulmonary toxicity (Zimetbaum 2012). The latter is a major cause for discontinuation of amiodarone treatment and has an incidence between 5 and $13 \%$. Amiodarone-induced pulmonary toxicity can occur acutely (within weeks after the start of therapy), in which case it is most commonly characterized by acute pulmonary hypersensitivity with patchy infiltrates, or develop with chronic treatment as increased pulmonary interstitial fibrosis (Schwaiblmair et al. 2010; Zimetbaum 2012). It has been suggested that amiodarone's iodine moieties and extreme lipophilic character strongly contribute to its toxicity. In addition, these properties result in a long half-life, such that side effects may persist (or even develop) after amiodarone treatment has been halted (Papiris et al. 2010). The pronounced toxicity of amiodarone has prompted the development of the derivative dronedarone, which lacks the iodine moieties and is less lipophilic than amiodarone, resulting in a much shorter half-life, although accumulation in tissues still occurs (Dobrev and Nattel 2010). In the DIONYSOS trial, dronedarone indeed resulted in a lower incidence of adverse thyroid, neurologic, skin, and ocular effects in patients with atrial fibrillation compared to amiodarone, although it was also less effective (Dobrev and Nattel 2010). However, more recent investigations have suggested that dronedarone does not have a superior safety profile compared to other antiarrhythmic drugs for the treatment of atrial fibrillation (Chatterjee et al. 2012; Said et al. 2012). In addition, the PALLAS trial was recently halted due to excess mortality in the dronedarone-treated arm, raising concerns about the usefulness of dronedarone (Nattel 2011). Other amiodarone derivatives with improved water solubility (e.g., budiodarone) are being developed and have shown a promise as antiarrhythmic agents for both atrial and ventricular arrhythmias (Goegelein et al. 2011; Billman et al. 2012; Ezekowitz et al. 2012).

In the current issue of Naunyn-Schmiedeberg's Archives of Pharmacology, Polat et al. (2013) show that amiodarone has a protective effect on lung tissue in a rat model of cecal ligation 
and puncture (CLP)-induced sepsis, characterized by a systemic inflammatory response as a consequence of an infection. In this study, rats underwent CLP via an abdominal incision. Sham-operated rats also received laparotomy, and their ceca were manipulated but not ligated or punctured. Following the operation, rats received saline or low- or highdose amiodarone ( 25 and $50 \mathrm{mg} / \mathrm{kg}$, respectively). In the $16 \mathrm{~h}$ postsurgery, the CLP rats showed a strong inflammatory response, as indicated by increased serum levels of the inflammatory cytokines interleukin (IL)- $1 \beta$, IL- 6 , and tumor necrosis factor alpha (TNF- $\alpha$ ). In addition, lung tissue of CLP rats showed reduced levels of antioxidants (glutathione and superoxide dismutase) and increased 8-iso-prostaglandin F2 $\alpha$, indicating oxidative stress. Histopathological analysis of lung tissue from CLP rats revealed infiltration of inflammatory cells and necrotic regions. All of these pathological responses were significantly attenuated in a dose-dependent manner in rats treated with amiodarone (Polat et al. 2013). Interestingly, there was also a tendency towards a lower mortality over the 16-h follow-up period in the amiodaronetreated groups. These results suggest that amiodarone may exert remarkable protective effects in pulmonary tissue in this model, in agreement with a previous study from the same authors in rats with carrageenan-induced paw edema (Halici et al. 2007). This striking protective action clearly contrasts to the pulmonary toxicity frequently observed in patients.

This study raises several important questions. Besides potential species differences, these seemingly paradoxical results likely depend on differences in dose and duration of amiodarone treatment. Indeed, amiodarone maintenance doses of more than $500 \mathrm{mg} /$ day have been shown to be more toxic than lower doses in the clinical setting, although pulmonary toxicity appears to develop with any dose (Papiris et al. 2010; Schwaiblmair et al. 2010). Moreover, in patients, there is an increased incidence of amiodarone-induced pulmonary toxicity with longer durations of treatment (Papiris et al. 2010; Schwaiblmair et al. 2010). In rats, the experimental animal of choice in the study by Polat et al. (2013), amiodarone has been associated with pulmonary toxicity after a period of several weeks (Reasor and Kacew 1996). Important contributing factors to amiodarone-induced pulmonary toxicity include direct cytotoxic effects (notably of $\mathrm{N}$-desethylamiodarone, the main metabolite of amiodarone), phopholipidosis, immunological mechanisms, and activation of the angiotensin system (reviewed in Papiris et al. 2010; Reasor and Kacew 1996). However, the exact mechanisms contributing to amiodaroneinduced pulmonary toxicity remain incompletely understood.

Many years of research on amiodarone's mechanisms of action has produced a complicated picture, showing a wide range of pleiotropic effects, both in the heart and elsewhere. Amiodarone and its active metabolite $N$-desethylamiodarone directly inhibit the cardiac $\mathrm{Na}^{+}$current $\left(I_{\mathrm{Na}}\right)$, a wide range of cardiac $\mathrm{K}^{+}$currents; the L-type $\mathrm{Ca}^{2+}$ current $\left(I_{\mathrm{Ca}, \mathrm{L}}\right)$; and the hyperpolarization-activated "funny current" $\left(I_{\mathrm{f}}\right)$, resulting in a reduction of cardiac excitability and prolongation of cardiac repolarization (reviewed in Heijman et al. 2013). In addition, amiodarone is a non-competitive $\alpha$ - and $\beta$-adrenoceptor antagonist. The direct electrophysiological effects of amiodarone and its derivative dronedarone are more pronounced in the atria than ventricles, likely contributing to their success in the treatment of atrial fibrillation and low incidence of ventricular proarrhythmia (Bogdan et al. 2011; Dobrev et al. 2012; Ehrlich and Dobrev 2011; Schmidt et al. 2012).

In contrast to other antiarrhythmic drugs, amiodarone can prevent atrial fibrillation-promoting atrial electrical remodeling in a dog model of pacing-induced atrial tachycardia, thereby reducing the incidence and duration of atrial fibrillation (Shinagawa et al. 2003). It has been suggested that prevention of L-type $\mathrm{Ca}^{2+}$ current $\alpha_{1 \mathrm{C}}$-subunit downregulation contributes to these anti-remodeling properties (Shinagawa et al. 2003). Similarly, amiodarone prevented both electrical and structural remodeling in the ventricles of canines with heart failure (Ashikaga et al. 2006) and in a rat model of inflammatory cardiomyopathy (Tachikawa et al. 2005). Of note, in the latter study, inflammatory markers IL- 6 and TNF- $\alpha$ were unchanged in myocardial tissue by treatment with $50 \mathrm{mg} / \mathrm{kg}$ amiodarone for 6 weeks (Tachikawa et al. 2005), in contrast to short-term treatment in the sepsis model (Polat et al. 2013), further highlighting the complex actions of amiodarone and the importance of differences in treatment duration and/or pathology.

The antioxidant and anti-inflammatory actions of amiodarone observed in the present study by Polat et al. (2013) are consistent with its effects on IL-1 $\beta$, IL-6, and TNF- $\alpha$ reported in human peripheral blood mononuclear cells after $24 \mathrm{~h}$ of culture in the presence of amiodarone (Matsumori et al. 1997), its effects in a mouse model of viral myocarditis (Ito et al. 2002), and its protective effects on canine ventricular myocytes during an acute challenge with $\mathrm{H}_{2} \mathrm{O}_{2}$ (Ide et al. 1999). However, chronic treatment with amiodarone (1 year) resulted in an increase in TNF- $\alpha$ in patients with ischemic cardiomyopathy, and no change in patients with nonischemic cardiomyopathy (Oral et al. 1999), again suggesting important differences between acute and long-term treatment.

Many additional effects of amiodarone have been described. For example, amiodarone and $\mathrm{N}$-desethylamiodarone are also potent vasodilators, affecting $\mathrm{Ca}^{2+}$ handling in endothelial cells (Grossmann et al. 1998, 2000; Himmel et al. 2000). The high atrial rate during atrial fibrillation induces oxidative stress and microvascular flow abnormalities in ventricles (Goette et al. 2009), and the acute anti-inflammatory and vasodilatory properties of amiodarone could contribute to its antiarrhythmic efficacy. Amiodarone and dronedarone have also emerged as potential treatments for Chagas disease, a tropical parasitic disease which is also associated with 
cardiovascular complications, notably dilated cardiomyopathy and associated arrhythmias (Benaim and Paniz Mondolfi 2012).

Taken together, these studies emphasize that half a century after its initial development, our mechanistic understanding of the pleiotropic actions of the most commonly prescribed antiarrhythmic drug is still incomplete. Although amiodarone at first glance appears to be an unlikely candidate for the protection of lung tissue during sepsis, given its well-known pulmonary toxicity, the work by Polat et al. (2013) shows that with the right dose and timing, the nonelectrophysiological actions may still confer important antioxidant and anti-inflammatory benefits. It would be interesting to determine in future studies whether similar benefits can be observed when sepsis is already present (i.e., during treatment as opposed to prevention), and whether improved survival is maintained during longer follow-up periods. A better understanding of the pleiotropic actions of amiodarone, combined with more detailed information on pathology-specific disease mechanisms, will likely facilitate the development of new pharmacological agents that can exploit the desirable effects while reducing the number of unwanted side effects.

Acknowledgments The authors' work is supported by the European Network for Translational Research in Atrial Fibrillation (EUTRAF), the German Federal Ministry of Education and Research [AF Competence Network and DZHK (German Center for Cardiovascular Research)], and the Deutsche Forschungsgemeinschaft (Do 769/1-3), and by a grant from Fondation Leducq (European-North American Atrial Fibrillation Research Alliance, 07CVD03).

Conflict of interest None.

\section{References}

Ashikaga K, Kobayashi T, Kimura M, Owada S, Sasaki S, Iwasa A, Furukawa K, Motomura S, Okumura K (2006) Effects of amiodarone on electrical and structural remodeling induced in a canine rapid pacing-induced persistent atrial fibrillation model. Eur J Pharmacol 536(1-2):148-153

Benaim G, Paniz Mondolfi AE (2012) The emerging role of amiodarone and dronedarone in Chagas disease. Nat Rev Cardiol 9(10):605-609

Billman GE, Gogelein H, Ruetten H, Wirth KJ (2012) Effects of a novel amiodarone-like compound SAR114646A on the pig atrium and susceptibility to ventricular fibrillation in dogs and pigs. Naunyn Schmiedebergs Arch Pharmacol 385(4):373-384

Bogdan R, Goegelein H, Ruetten H (2011) Effect of dronedarone on $\mathrm{Na}^{+}, \mathrm{Ca}^{2+}$ and HCN channels. Naunyn Schmiedebergs Arch Pharmacol 383(4):347-356

Chatterjee S, Ghosh J, Lichstein E, Aikat S, Mukherjee D (2012) Metaanalysis of cardiovascular outcomes with dronedarone in patients with atrial fibrillation or heart failure. Am J Cardiol 110(4):607613

Dobrev D, Nattel S (2010) New antiarrhythmic drugs for treatment of atrial fibrillation. Lancet 375(9721):1212-1223
Dobrev D, Carlsson L, Nattel S (2012) Novel molecular targets for atrial fibrillation therapy. Nat Rev Drug Discov 11(4):275-291

Echt DS, Liebson PR, Mitchell LB, Peters RW, Obias-Manno D, Barker AH, Arensberg D, Baker A, Friedman L, Greene HL et al (1991) Mortality and morbidity in patients receiving encainide, flecainide, or placebo. The Cardiac Arrhythmia Suppression Trial. N Engl J Med 324(12):781-788

Ehrlich JR, Dobrev D (2011) Atrial-selective sodium channel block by dronedarone: sufficient to terminate atrial fibrillation? Naunyn Schmiedebergs. Arch Pharmacol 384(2):109-114. doi:10.1007/ s00210-011-0647-5

Ezekowitz MD, Nagarakanti R, Lubinski A, Bandman O, Canafax D, Ellis DJ, Milner PG, Ziola M, Thibault B, Hohnloser SH, Investigators $\mathrm{P}$ (2012) A randomized trial of budiodarone in paroxysmal atrial fibrillation. J Interv Card Electrophysiol 34(1):1-9

Goegelein H, Gautier P, Roccon A, O'Connor S, Ruetten H (2011) Effects of the novel amiodarone-like compound SAR114646A on cardiac ion channels and ventricular arrhythmias in rats. Naunyn Schmiedebergs. Arch Pharmacol 384(3):231-244

Goette A, Bukowska A, Dobrev D, Pfeiffenberger J, Morawietz H, Strugala D, Wiswedel I, Rohl FW, Wolke C, Bergmann S, Bramlage P, Ravens U, Lendeckel U (2009) Acute atrial tachyarrhythmia induces angiotensin II type 1 receptor-mediated oxidative stress and microvascular flow abnormalities in the ventricles. Eur Heart J 30(11):1411-1420

Grossmann M, Dobrev D, Kirch W (1998) Amiodarone causes endothelium-dependent vasodilation in human hand veins in vivo. Clin Pharmacol Ther 64(3):302-311

Grossmann M, Dobrev D, Himmel HM, Kirch W (2000) Local venous response to $N$-desethylamiodarone in humans. Clin Pharmacol Ther 67(1):22-31

Halici Z, Dengiz GO, Odabasoglu F, Suleyman H, Cadirci E, Halici M (2007) Amiodarone has anti-inflammatory and anti-oxidative properties: an experimental study in rats with carrageenaninduced paw edema. Eur J Pharmacol 566(1-3):215-221

Heijman J, Voigt N, Dobrev D (2013) New directions in antiarrhythmic drug therapy for atrial fibrillation. Future Cardiol 9(1):71-88

Himmel HM, Dobrev D, Grossmann M, Ravens U (2000) Ndesethylamiodarone modulates intracellular calcium concentration in endothelial cells. Naunyn Schmiedebergs. Arch Pharmacol 362(6):489-496

Ide T, Tsutsui H, Kinugawa S, Utsumi H, Takeshita A (1999) Amiodarone protects cardiac myocytes against oxidative injury by its free radical scavenging action. Circulation 100(7):690-692

Ito H, Ono K, Nishio R, Sasayama S, Matsumori A (2002) Amiodarone inhibits interleukin 6 production and attenuates myocardial injury induced by viral myocarditis in mice. Cytokine 17(4):197-202

Matsumori A, Ono K, Nishio R, Nose Y, Sasayama S (1997) Amiodarone inhibits production of tumor necrosis factor-alpha by human mononuclear cells: a possible mechanism for its effect in heart failure. Circulation 96(5):1386-1389

Nattel S (2011) Dronedarone in atrial fibrillation-Jekyll and Hyde? N Engl J Med 365(24):2321-2322

Oral H, Fisher SG, Fay WP, Singh SN, Fletcher RD, Morady F (1999) Effects of amiodarone on tumor necrosis factor-alpha levels in congestive heart failure secondary to ischemic or idiopathic dilated cardiomyopathy. Am J Cardiol 83(3):388-391

Papiris SA, Triantafillidou C, Kolilekas L, Markoulaki D, Manali ED (2010) Amiodarone: review of pulmonary effects and toxicity. Drug Saf 33(7):539-558

Polat B, Cadirici E, Halici Z, Bayir Y, Unal D, Bilgin BC, Yuksel TN, Vancelik S (2013) The protective effect of amiodarone in lung tissue of cecal ligation and puncture-induced septic rats: a perspective from inflammatory cytokine release and oxidative stress. Naunyn Schmiedebergs Arch Pharmacol (in press). 
Reasor MJ, Kacew S (1996) An evaluation of possible mechanisms underlying amiodarone-induced pulmonary toxicity. Proc Soc Exp Biol Med 212(4):297-304

Said SM, Esperer HD, Kluba K, Genz C, Wiedemann AK, Boenigk H, Herold J, Schmeisser A, Braun-Dullaeus RC (2012) Efficacy and safety profile of dronedarone in clinical practice. Results of the Magdeburg Dronedarone Registry (MADRE study). Int J Cardiol (in press)

Schmidt C, Wiedmann F, Schweizer PA, Becker R, Katus HA, Thomas D (2012) Novel electrophysiological properties of dronedarone: inhibition of human cardiac two-pore-domain potassium (K2P) channels. Naunyn Schmiedebergs. Arch Pharmacol 385(10):10031016

Schwaiblmair M, Berghaus T, Haeckel T, Wagner T, von Scheidt W (2010) Amiodarone-induced pulmonary toxicity: an underrecognized and severe adverse effect? Clin Res Cardiol 99(11):693-700

Shinagawa K, Shiroshita-Takeshita A, Schram G, Nattel S (2003) Effects of antiarrhythmic drugs on fibrillation in the remodeled atrium: insights into the mechanism of the superior efficacy of amiodarone. Circulation 107(10):1440-1446

Singh BN (2008) Amiodarone as paradigm for developing new drugs for atrial fibrillation. J Cardiovasc Pharmacol 52(4):300-305

Singh BN, Vaughan Williams EM (1970) The effect of amiodarone, a new anti-anginal drug, on cardiac muscle. Br J Pharmacol 39(4):657-667

Tachikawa H, Kodama M, Watanabe K, Takahashi T, Ma M, Kashimura T, Ito M, Hirono S, Okura Y, Kato K, Hanawa H, Aizawa Y (2005) Amiodarone improves cardiac sympathetic nerve function to hold norepinephrine in the heart, prevents left ventricular remodeling, and improves cardiac function in rat dilated cardiomyopathy. Circulation 111(7):894-899

Waldo AL, Camm AJ, deRuyter H, Friedman PL, MacNeil DJ, Pauls JF, Pitt B, Pratt CM, Schwartz PJ, Veltri EP (1996) Effect of d-sotalol on mortality in patients with left ventricular dysfunction after recent and remote myocardial infarction. The SWORD Investigators. Survival With Oral d-Sotalol. Lancet 348(9019):7-12

Zimetbaum P (2012) Antiarrhythmic drug therapy for atrial fibrillation. Circulation 125(2):381-389 